\title{
Micro-local energy method of Mizohata and hypoellipticity
}

\author{
By
}

Toshihiko HosHiro

\section{§. Introduction}

In the present paper, we are mainly concerned with partial hypoellipticity (with respect to the $x$-variables) of the equation (in $\mathbf{R}^{d^{+1}}$ )

$$
\frac{\partial u}{\partial t}+a\left(x, D_{x}\right) u=f
$$

where $a\left(x, D_{x}\right)$ is a second order partial differential operator (in $\mathbf{R}^{d}$ with coefficients of class $\left.\mathrm{C}^{\infty}\right)$ satisfying the following conditions.

There exists a constant $C$ such that

$$
\begin{aligned}
& \sum_{|\nu|=1}\left\|a^{(\nu)}\left(x, D_{x}\right) v\right\|^{2}+\sum_{|\mu|=1}\left\|a_{(\mu)}\left(x, D_{x}\right) v\right\|_{-1}^{2} \\
& \quad \leqq C\left(\operatorname{Re}\left(a\left(x, D_{x}\right) v, v\right)+\|v\|^{2}\right), \quad \forall v \in \mathscr{S}\left(\boldsymbol{R}^{d}\right) .
\end{aligned}
$$

For any $\varepsilon>0$, there exists a constant $C_{\varepsilon}$ such that

$$
\left\|\log \left\langle D_{x}\right\rangle v\right\|^{2} \leqq \varepsilon \operatorname{Re}\left(a\left(x, D_{x}\right) v, v\right)+C_{\varepsilon}\|v\|^{2}, \forall v \in \mathscr{S}\left(\boldsymbol{R}^{d}\right) .
$$

Here we use standard notations: $a_{(\mu)}^{(\nu)}(x, \xi)=\partial_{\xi}^{\nu}\left(-i \partial_{x}\right)^{\mu} a(x, \xi)$, and $\log \left\langle D_{x}\right\rangle=\log$ $\left(2-\Delta_{x}\right)^{1 / 2}$. \|\| and \|\|$_{-1}$ stand for the norms in $L^{2}\left(\boldsymbol{R}^{d}\right)$ and $H^{-1}\left(\boldsymbol{R}^{d}\right)$ (the Sobolev space of order -1 ) respectively.

Our main purpose is:

Theorem 1. Assume that second order differential operator $a\left(x, D_{x}\right)$ satisfies the conditions (A.1) and (A.2). Suppose that $u \in C^{1}\left([0, \delta] ; \mathscr{D}_{x}^{\prime}(U)\right)$ and $f \in C([0, \delta]$; $\left.\mathscr{D}_{x}^{\prime}(U)\right)$ satisfy the eguation $(0.1)$ in $(0, \delta) \times U$, where $\delta>0$ and $U$ is an open set of $\boldsymbol{R}^{d}$. Let $\left(x_{0}, \xi^{0}\right)$ be a point of $U \times\left(\boldsymbol{R}^{d} \backslash 0\right)$. Then, if $\left(x_{0}, \xi^{0}\right) \notin W F(f(\cdot, t))$ for $0 \leqq t \leqq \delta$, it follows that $\left(x_{0}, \xi^{0}\right) \notin W F(u(\cdot, t))$ for $0<t \leqq \delta$.

Since $a u(x)=f(x)$ implies $\partial u / \partial t+a u=f$ (where $u$ and $f$ are independent of $t$ ), Theorem 1 yields the result due to Y. Morimoto [7]:

Corollary. Provided second order differential operator $a\left(x, D_{x}\right)$ satisfies the conditions (A.1) and (A.2), then it follows that $a\left(x, D_{x}\right)$ is hypoelliptic in $\boldsymbol{R}^{d}$.

Our argument can be applied without modification to the proof of the follow- 
ing Morimoto's theorem:

Theorem 2. (Y. Morimoto [8]). Let $a\left(x, D_{x}\right)$ be a differential operator of order $m$ with coefficients of class $C^{\infty}\left(\boldsymbol{R}^{d}\right)$. Assume that for any $\varepsilon>0$, there exists a constant $C_{\varepsilon}$ such that

$$
\begin{aligned}
& \left\|\left(\log \left\langle D_{x}\right\rangle\right)^{m} v\right\|^{2}+\sum_{0<|\nu+\mu|<m}\left\|\left(\log \left\langle D_{x}\right\rangle\right)^{|\nu+\mu|} a_{(\mu)}^{(\nu)}\left(x, D_{x}\right) v\right\|^{2}-|\mu| \\
& \quad \leqq \varepsilon\left\|a\left(x, D_{X}\right) v\right\|^{2}+C_{\varepsilon}\|v\|^{2}, \quad \forall v \in \mathscr{S}\left(\boldsymbol{R}^{d}\right) .
\end{aligned}
$$

Then, $a\left(x, D_{x}\right)$ is hypoelliptic. Moreover we have $W F(a u)=W F(u)$ for all $u \in \mathscr{D}^{\prime}\left(\boldsymbol{R}^{d}\right)$.

Let us now explain the conditions (A.1) and (A.2). To the operators of the form $a\left(x, D_{x}\right)=\sum_{j=1}^{k} X_{j}^{*} X_{j}\left(o r=\sum_{j=1}^{k} X_{j}^{2}\right)$, where $X_{j}, j=1, \ldots, k$ are C co $^{\infty}$ real vector fields generating a Lie algebra of rank $d$ at every point, Theorem 1 is applicable. In fact, the condition (A.1) is satisfied by the operators of the form $\sum_{j=1}^{k} X_{j}^{2}+X_{0}\left(X_{j}\right.$, $j=0, \ldots, k$ are real vector fields of class $\left.\mathrm{C}^{\infty}\right)$, and, provided $X_{1}, \ldots, X_{k}$ satisfy the condition on Lie algebra, the operators of the form $a\left(x, D_{x}\right)=\sum_{j=1}^{k} X_{j}^{*} X_{j}$ (or $\left.=\sum_{j=1}^{k} X_{j}^{2}\right)$ have

$$
\left\|\langle D\rangle^{\sigma} v\right\|^{2} \leqq \text { const. }\left(\operatorname{Re}\left(a\left(x, D_{x}\right) v, v\right)+\|v\|^{2}\right), \forall v \in \mathscr{S}\left(\boldsymbol{R}^{d}\right)
$$

for some $\sigma, 0<\sigma \leqq 1$. (See L. Hörmander [1] chap. 22.2.) Theorem 1 is also applicable to the infinitely degenerate elliptic operators (they are our main targets). For example, the operator

$$
a_{1}\left(x, D_{x}\right)=D_{x_{1}}^{2}+x_{1}^{2 k} D_{x_{2}}^{2}+\exp \left(-1 /\left|\mathrm{x}_{2}\right|^{\gamma}\right) D_{x_{3}}^{2}
$$

satisfies (A.1) and (A.2), if $k$ is a non-negative integer and $0<\gamma<\frac{1}{k+1}$. (See Y. Morimoto [7] proposition 4.)

On the other hand, it is remarkable that the condition (A.2) is necessary for hypoellipticity of the operators of the form $a\left(x, D_{x}\right)=D_{x_{1}}^{2}+b\left(x^{\prime}, D_{x^{\prime}}\right)$, where $b\left(x^{\prime}, D_{x^{\prime}}\right)$ is a formally selfadjoint differential operator of second order satisfying $\left(b\left(x^{\prime}, D_{x^{\prime}}\right) v, v\right) \geqq-$ const. $\|v\|^{2}, \forall v \in \mathscr{S}\left(\boldsymbol{R}^{d-1}\right)$. (From this fact, we can show that the operator $a_{1}\left(x, D_{X}\right)$ with $k=0$ is not hypoelliptic in any neighborhood of the origin $0 \in \boldsymbol{R}^{d}$, if $\gamma \geqq 1$. See Y. Morimoto [7] or T. Hoshiro [2].)

We rely heavily on the works [4] and [5] by S. Mizohata, who initiated the micro-local energy method for the characterizations of the analytic and the Gevrey wave front sets. We remark that our method is quite elementary. In fact, we make use of basic calculus of pseudo-differential operators.

Hypoellipticity of $a\left(x, D_{x}\right)$ under the conditions (A.1) and (A.2) (or under the condition (A.3)) was recently studied by Y. Morimoto, related to the work by S. Kusuoka - D. Strook [3]. He has obtained the corollary to Theorem 1 and also Theorem 2 in [7] and [8] respectively. Essentially, our results are not new. However, we believe that our method is one of the most direct way to arrive at the results.

The plan of this paper is as follows: In $\S 1$, we explain our method. The 
proof of Theorem 1 will be given in $\$ 2$. In $\$ 3$, we will prove the propositions stated in $\$ 1$. Finally in $\S 4$, we will prove Theorem 2.

Acknowledgments. The author is deeply indebted to Professors S. Mizohata, W. Mastumoto and N. Shimakura for helpful encouragements.

\section{$\S 1$. Preliminaries.}

In this section, we introduce some notations and techniques which are necessary for the proofs of Theorems. Let us first define a sequence of cut-off functions (micro-localizers) $\left\{\alpha_{n}(\xi), \beta_{n}(x)\right\}_{n \geqq 1}$, of size $r_{0}>0$. It is well known that there exists a sequence $\phi_{N} \in C_{0}^{\infty}\left(\boldsymbol{R}^{d}\right), N=1,2, \ldots$, having the following properties:

(C-1) $\phi_{N}$ has its support in $\left\{y ;|y| \leqq r_{0}\right\}$, and is equal to 1 in $\left\{y ;|y| \leqq r_{0} / 2\right\}$.

For any positive integer $K_{0}$,

$$
\begin{aligned}
& \left|\partial_{y}^{p+\nu} \psi_{N}\right| \leqq C_{K_{0}}(C N)^{|p|} \\
& |p| \leqq N \text { and }|\nu| \leqq K_{0}
\end{aligned}
$$

where $C$ and $C_{K_{0}}$ are constants independent of $N$.

(For the construction of $\phi_{N}$, see S. Mizohata [5].)

Now we define

$$
N_{n}=[\log n]+1, \alpha_{n}(\xi)=\psi_{N_{n}}\left(\frac{\xi}{n}-\xi^{0}\right) \text { and } \beta_{n}(x)=\phi_{N_{n}}\left(x-x_{0}\right) .
$$

Note: Let us notice that the support of $\alpha_{n}(\xi)$ is contained in $\left\{\xi ;\left|\xi-n \xi^{0}\right| \leqq n r_{0}\right\}$, in other words, since $r_{0}$ is small, the support of $\alpha_{n}(\xi)$ is contained in a small conic neighborhood of $\xi^{0}$. We remark that, in the following arguments, we are mainly concerned with the investigations when $n$ is large. So we may regard $N_{n}$ as $\log n$. Also we remark that, in our method, (C-2) is very important because we have

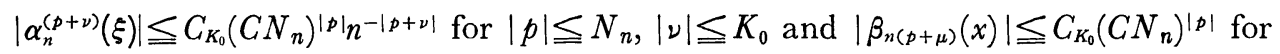
$|q| \leqq N_{n},|\mu| \leqq K_{0}$, which enable us to do the quantitative analysis. $\left(\alpha_{n}^{(p+\nu)}=\partial_{\xi}^{p+\nu} \alpha_{n}\right.$, $\beta_{n(q+\mu)}=\left(-i \partial_{x}\right)^{p+\mu} \beta_{n}$.)

Let us put, for $u \in \mathscr{D}^{\prime}\left(\mathbf{R}^{d}\right)$,

$$
\left\{\begin{array}{l}
S_{n}^{M}(u)=\sum_{|p+q| \leqq N_{n}}\left\|c_{p, q}^{n} \alpha_{n}^{(p)}(D) \beta_{n(q)}(x) u\right\|^{2} \\
\text { with } c_{p, q}^{n}=M^{-|p+q|} n^{|p|}(\log n)^{-|p+q|} .
\end{array}\right.
$$

$S_{n}^{M}(u)$ could be called a micro-local energy of $u$ in a neighborhood of $\left(x_{0}, n \xi^{0}\right)$. Now, we have the following propositions on the relation between the wave front sets and behavior of $S_{n}^{M}(u)$ as $n \rightarrow \infty$, whose proofs will be given in $\S 3$.

Proposition 1. Let $u$ be a distribution and suppose $\left(x_{0}, \xi^{0}\right) \notin W F(u)$. Then we have, if $r_{0}$ is small: 


$$
\left\{\begin{array}{c}
\text { For any positive number } s \text {, there exists a } M>0 \text { such that } \\
\qquad S_{n}^{M}(u)=O\left(n^{-2 s}\right) \text { as } n \rightarrow \infty .
\end{array}\right.
$$

Note: In general, $\phi_{n}=O\left(n^{-k}\right)$ means that there exists a constant $B$ such that $\left|\phi_{n}\right| \leqq B n^{-k}$, when $n$ is large.

The converse is also true. We can state in the following form:

Proposition 2. Put:

$$
\tilde{S}_{n}^{M}(u)=\sum_{|p| \leqq N_{n}}\left\|c_{p, 0}^{n} \alpha_{n}^{(p)}(D) \beta_{n}(x) u\right\|^{2} .
$$

If $\tilde{S}_{n}^{M}(u)$ satisfies (1.3), then $\left(x_{0}, \xi^{0}\right) \notin W F(u)$.

Note: It follows from Proposition 1 and 2 that $\left(x_{0}, \xi^{0}\right) \notin W F(u)$ is equivalent to (1.3) because $S_{n}^{M}(u) \leqq S_{n}^{M}(u)$. Also notice that $S_{n}^{M}(u) \leqq S_{n}^{M^{\prime}}(u)$ if $M \geqq M^{\prime}$.

Let us notice the following fact. In the definition (1.2), if we take $M$ sufficiently large, the values of $\left\|c_{p, q}^{n} \alpha_{n}^{(p)} \beta_{n(q)} u\right\|,(p, q)$ satisfying $|p+q|=N_{n}$, are so small that we can neglect them, when $n$ is large. More precisely,

Lemma 1. Let $u$ be a distribution. For any positive number s, if we choose $M$ sufficiently large, then

$$
\begin{aligned}
\stackrel{\circ}{S}_{n}^{M}(u) & =\sum_{|p+q|=N_{n}}\left\|c_{p, q}^{n} \alpha_{n}^{(p)}(D) \beta_{n(q)}(x) u\right\|^{2} \\
& =O\left(n^{-2 s}\right) \text { as } n^{n \rightarrow \infty} .
\end{aligned}
$$

In the sequel, we call a term (or sum of terms) negligible if it satisfies the same property as $S_{n}^{M}(u)$ in Lemma 1. By the same argument as in Proof of Lemma 1, we can show that the sum of $\left\|c_{p, q}^{n} \alpha_{n}^{(p)} \beta_{n(q)} u\right\|^{2}$ with respect to $(p, q)$ satisfying $N_{n}-n_{0} \leqq|p+q| \leqq N_{n}, n_{0}$ being any fixed positive integer independent of $n$, is also negligible.

Proof of Lemma 1. First, we take a $\zeta \in C_{0}^{\infty}$ so that $\zeta(x)=1$ in $\left\{x ;\left|x-x_{0}\right|\right.$ $\left.\leqq r_{0}\right\}$. (Therefore $\beta_{n} \subset \subset \zeta$.) Then, for some $k$, we have $\zeta u \in H^{-k}$. Let us now recall that, for $c(x, \xi) \in S_{1,0}^{-k}$, the semi-norm of $c(x, \xi)$ in $S_{1,0}^{-k}$ is defined in such a way that

$$
|c|_{l}^{(-k)}=\max _{|\nu+\mu| \leqq l} \sup \left|c_{(\nu)}^{(\mu)}(x, \xi)\right| /\langle\xi\rangle^{-k-|\mu|} .
$$

From the condition (C-2) and fact that $K^{-1} n \leqq \sqrt{1+|\xi|^{2}} \leqq K \cdot n$ for $\xi \in \operatorname{supp}\left[\alpha_{n}^{(p)}\right]$, it follows that the semi-norms (in $\left.S_{1,0}^{-k}\right)$ of symbols $\sigma\left(c_{p, q}^{n} \alpha_{n}^{(p)} \beta_{n(q)}\right),(p, q)$ satisfying $|p+q| \leqq N_{n}$, are estimated by $n^{k} \cdot C^{\prime}(2 C \mid M)^{|p+q|}$, where $C$ (resp. $M$ ) is the same constant as in (C-2) (resp. (1.2)). Therefore, the values of

$$
\left\|c_{p, q}^{n} \alpha_{n}^{(p)}(D) \beta_{n(q)}(x)\left\langle D_{x}\right\rangle k\right\|_{L^{2} \rightarrow L^{2}},
$$

$(p, q)$ satisfying $|p+q| \leqq N_{n}$, are also estimated by $n^{k} \cdot C^{\prime \prime}(2 C / M)^{|p+q|}$.

To see these estimates, observe that 


$$
\begin{aligned}
\left|\sigma\left(\alpha_{n}^{(p)} \beta_{n(q)}\right)\right|_{l}^{(-k)} & \leqq \text { const. }\left|\alpha_{n}^{(p)}\right|_{l_{1}}^{(-k)}\left|\beta_{n(q)}\right|_{l_{1}}^{(0)} \\
& \leqq \text { const. } n^{k-|p|}\left(C N_{n}\right)^{|p|}\left(C N_{n}\right)^{|q|}
\end{aligned}
$$

where const.s are independent of $n, p$ and $q$ (we take $K_{0}=l_{1}$ in (C-2)). We choose $l=d+2$ in order to apply (1.4) to the values of $\left\|c_{p, q}^{n} \alpha_{n}^{(p)} \beta_{n(q)}\left\langle D_{x}\right\rangle k\right\|_{L^{2} \rightarrow L^{2}}$, $(p, q)$ satisfying $|p+q| \leqq N_{n}$.

On the other hand, the number of terms with $(p, q)$ satisfying $|p+q|=N_{n}$ cannot exceed $(2 d)^{N_{n}}$. Therefore we have

$$
\begin{aligned}
\stackrel{\circ}{S}_{n}^{M}(u) & =\sum_{|p+q|=N_{n}} \| c_{p, q}^{n} \alpha_{n}^{(p)} \beta_{n(q)}\left\langle D_{x}\right\rangle\left\langle\left\langle D_{x}\right\rangle^{-k \zeta u \|^{2}}\right. \\
& \leqq C_{1} \sum_{|p+q|=N_{n}}\left\|c_{p, q}^{n} \alpha_{n}^{(p)} \beta_{n(q)}\left\langle D_{x}\right\rangle\right\|_{L^{2} \rightarrow L^{2}}^{2} \\
& \leqq C_{2} \cdot n^{2 k} \cdot(2 C / M)^{2 N_{n}} \cdot(2 d)^{N_{n}},
\end{aligned}
$$

where $C_{j}, j=1,2$, are constants independent of $n$.

Now, for the given positive number $s$, we choose $M$ in such a way that $(2 C / M) \cdot \sqrt{2 d} \leqq e^{-s-k}$. Then the last inequality is estimated by const. $\cdot n^{-2 s}$ when $n$ is large (recall that $N_{n} \fallingdotseq \log n$ ).

Note: There are several choices of micro-local energy forms related to the criterion of the wave front sets (see S. Mizohara [4]). The reader might feel that our form $S_{n}^{M}(u)$ looks like strange because, in spite of the analysis in $C^{\infty}$-class, the sum on $|p+q|$ is taken up to $N_{n} \fallingdotseq \log n$ (which becomes large with $n$ ). However in our arguments, the choice, together with the choice of $c_{p, q}^{n}$ and the condition (C-2), is essential in view of the assumption (A.2).

\section{§. Proof of Theorem 1.}

Let us admit Proposition 1 and 2 for the moment. Our assumption for $f(x, t)$ is:

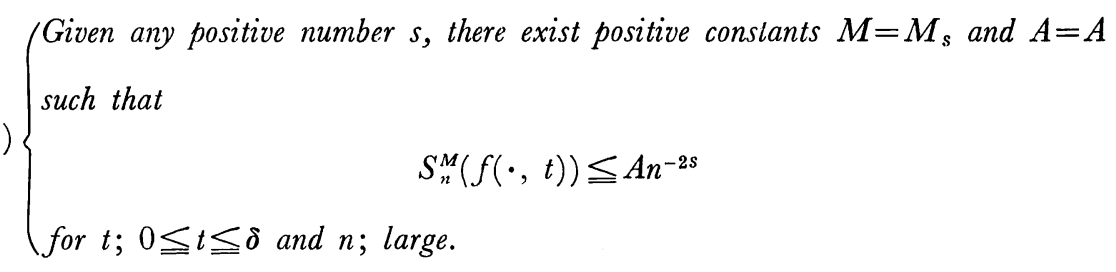

Under this assumption (for the given $s$ ), we are gcing to prove:

$$
\left\{\begin{array}{l}
\text { For any } \eta(0<\eta<\delta) \text {, there exists a } B=B(\eta, s) \text { such that } \\
\qquad S_{n}^{M}(u(\cdot, t)) \leqq B n^{-2 s} \\
\text { for } t ; \eta \leqq t \leqq \delta \text { and } n ; \text { large. }
\end{array}\right.
$$

We show this by the method of S. Mizohata [5]. Since the proof is long, we devide it into three steps. 
(Step 1.) First, we operate $\alpha_{n}^{(p)}(D) \beta_{n(q)}(x),(p, q)$ satisfying $|p+q| \leqq N_{n}$, to the both sides of the equation (0.1). Then

$$
\partial_{t} u_{p, q}=-\alpha_{n}^{(p)} \beta_{n(q)} a\left(x, D_{x}\right) u+f_{p, q},
$$

where we denote $u_{p, q}=\alpha_{n}^{(p)} \beta_{n(q)} u$ and $f_{p, q}=\alpha_{n}^{(p)} \beta_{n(q)} f$.

Therefore we have

$$
\begin{aligned}
\frac{d}{d t}\left\|u_{p, q}\right\|^{2}= & 2 \operatorname{Re}\left(-\alpha_{n}^{(p)} \beta_{n(q)} a\left(x, D_{x}\right) u+f_{p, q}, u_{p, q}\right)= \\
= & 2 \operatorname{Re}\left\{-\left(a\left(x, D_{x}\right) u_{p, q}, u_{p, q}\right)\right. \\
& +\sum_{|\nu|=1}\left(a^{(\nu)}\left(x, D_{x}\right) u_{p, q+\nu}, u_{p, q}\right)-\sum_{|\mu|=1}\left(a_{(\mu)}\left(x, D_{x}\right) u_{p+\mu, q}, u_{p, q}\right) \\
& -\sum_{2 \leqq} \sum_{\nu+\mu \mid \leqq N_{0}} \frac{(-1)^{|\nu|}}{\nu ! \mu !}\left(a_{(\mu)}^{(\nu)}\left(x, D_{x}\right) u_{p+\mu, q+\nu}, u_{p, q}\right) \\
& \left.-\left(r_{p, q}\left(x, D_{x}\right) u, u_{p, q}\right)+\left(f_{p, q}, u_{p, q}\right)\right\} \\
= & 2 \operatorname{Re} \sum_{j=1}^{6} I_{j},
\end{aligned}
$$

where $N_{0}$ is a large integer whose definition will be given later (see (2.4)).

(Step 2.) Now, we have the following:

Lemma 2. (i) The terms $r_{p, q}\left(x, D_{x}\right) u,(p, q)$ satisfying $|p+q| \leqq N_{n}$, are negligible, that is, if we take $N_{0}$ sufficiently large, then

$$
\sum_{|p+q| \leqq N_{n}}\left\|c_{p, q}^{n} r_{p, q}\left(x, D_{x}\right) u\right\|^{2}=0\left(n^{-2 s}\right) \text { as } n \rightarrow \infty .
$$

Here, $\phi_{n}(t)=0\left(n^{-k}\right)$ means that there exists a constant $B$ such that $\left|\phi_{n}(t)\right| \leqq B n^{-k}$, for $t$; $0 \leqq t \leqq \delta$ and $n$; large.

(ii) For every positive number $L$,

$$
\begin{aligned}
\left|I_{2}\right| \leqq & L^{-1}(\log n)^{-2} \text { const. } \sum_{|\nu|=1}\left\{\operatorname{Re}\left(a\left(x, D_{x}\right) u_{p, q+\nu}, u_{p, q+\nu}\right)\right. \\
& \left.+\left\|u_{p, q+\nu}\right\|^{2}\right\}+L(\log n)^{2}\left\|u_{p, q}\right\|^{2}
\end{aligned}
$$

and

$$
\begin{aligned}
\left|I_{3}\right| \leqq & L^{-1} n^{2}(\log n)^{-2} \text { const. } \sum_{|u|=1}\left\{\operatorname{Re}\left(a\left(x, D_{x}\right) u_{p+\mu, q}, u_{p+\mu, q}\right)\right. \\
& \left.+\left\|u_{p+\mu, q}\right\|^{2}\right\}+L(\log n)^{2}\left\|u_{p, q}\right\|^{2},
\end{aligned}
$$

where const.s are independent of $L, n, p$ and $q$.

(iii) For every positive number $L$,

$$
\begin{aligned}
&\left|I_{4}\right| \leqq L^{-1} \text { const. } \sum_{2 \leqq|\nu+\mu| \leqq N_{0}} n^{2(2-|\nu|)}(\log n)^{-4} \times \\
& \times\left\{\operatorname{Re}\left(a\left(x, D_{x}\right) u_{p+\mu, q+\nu}, u_{p+\mu, q+\nu}\right)+\left\|u_{p+\mu, q+\nu}\right\|^{2}\right\} \\
&+L(\log n)^{2}\left\|u_{p, q}\right\|,
\end{aligned}
$$


where const. is independent of $L, n, p$ and $q$.

Proof of Lemma 2. (i) Take a function $\zeta \in C_{0}^{\infty}(\boldsymbol{R} d)$ satisfying $\zeta(x)=1$ in $\left\{x ;\left|x-x_{0}\right| \leqq r_{0}\right\}$. Then, $\beta_{n} \subset \subset \zeta$ and there exists a positive number $k$ such that $\zeta u(\cdot, t) \in H^{-k}\left(\boldsymbol{R}^{d}\right)$ for $t ; 0 \leqq t \leqq \delta$.

Let us observe the followings. From the condition (C-2) and the fact that $K^{-1} n \leqq \sqrt{1+|\xi|^{2}} \leqq K \cdot n$ for $\xi \in \operatorname{supp}\left[\alpha_{n}^{(p)}\right]$, it follows that the semi-norms of $c_{p, q}^{n} r_{p, q}$ $\left(x, D_{x}\right)$ in $S_{1,0}^{s+1-N_{0}},(p, q)$ satisfying $|p+q| \leqq N_{n}$, are estimated by $n^{-s} C^{\prime}(2 C / M)^{|p+q|}$. Therefore the values of

$$
\left\|c_{p, q}^{n} r_{p, q}\left(x, D_{x}\right)\left\langle D_{x}\right\rangle^{k}\right\|_{L^{2} \rightarrow L^{2}},
$$

$(p, q)$ satisfying $p+q \mid \leqq N_{n}$, are also estimated by $n^{-s} C^{\prime \prime}(2 C / M)^{|p+q|}$, provided

$$
s+1-N_{0} \leqq-k \text {. }
$$

To see these estimates, observe that (cf. S. Mozohata [4] page 58)

$$
\begin{aligned}
& \left|r_{p, q}\right|_{l}^{\left(s+1-N_{0}\right)} \\
& \quad \leqq \text { const. } \sum_{|\nu+\mu|=N_{0}+1}\left|\alpha_{n}^{(p+\mu)}\right|_{l_{1}}^{(s-|\mu|)}\left|\beta_{n(q+\nu)}\right|_{l_{1}}^{(0)}\left|a_{(\mu)}^{(\nu)}\right| l_{l_{1}}^{(2-|\nu|)} \\
& \quad \leqq \text { const. } n^{-s-|p|}\left(C N_{n}\right)^{|p+q|},
\end{aligned}
$$

where const.s are constants independent of $n, p$ and $q$.

So, let us take $N_{0}$ in such a way that the inequality (2.4) holds. Then we have (notice that, in (2.3), $u$ may be replaced by $\zeta u$, because $a\left(x, D_{x}\right)$ is a differential operator and $\left.\beta_{n} \subset \subset \zeta\right)$

$$
\begin{aligned}
& \sum_{|p+q| \leqq N_{n}}\left\|c_{p, q}^{n} r_{p, q}\left(x, D_{x}\right) u\right\|^{2} \\
& =\sum_{|p+q| \leqq N_{n}} \| c_{p, q}^{n} r_{p, q}\left(x, D_{x}\right)\left\langle D_{x}\right\rangle\left\langle D_{x}\right\rangle^{-k \zeta u \|^{2}} \\
& \quad \leqq C_{|p+q| \leqq N_{n}} \mid c_{p, q}^{n} r_{p, q}\left(x, D_{x}\right)\left\langle D_{x}\right\rangle k \|_{L^{2} \rightarrow L^{2}}^{2} \\
& \leqq C_{2} \sum_{p, q}(2 C / M)^{2|p+q| n^{-2 s}} \\
& \leqq C_{2} n^{-2 s}
\end{aligned}
$$

provided $M$ satisfies $2 d(2 C / M)^{2}<1 / 2$. (Observe that the number of the terms with $(p, q)$ satisfying $|p+q|=j$ cannot exceed $(2 d)^{j}$.)

(ii) Let us show the second inequality, because the proof of the first one is similar. First, it is clear that

$$
\left|I_{3}\right| \leqq L^{-1} d(\log n)^{-2} \sum_{|\mu|=1}\left\|a_{(\mu)} u_{p+\mu, q}\right\|^{2}+L(\log n)^{2}\left\|u_{p, q}\right\|^{2} .
$$

Let us take a function $\phi \in C_{0}^{\infty}$ so that $\phi(y)=1$ in $\left\{y ;|y| \leqq r_{0}\right\}$, and define $\tilde{\alpha}_{n}(\xi)=\phi\left(\frac{\xi}{n}-\xi^{0}\right)$. Then, since $\alpha_{n} \subset \subset \tilde{\alpha}_{n}$ and the commutator $\left[\tilde{\alpha}_{n}, a_{(\mu)}\right]$ is bounded in $O P S_{1,0}^{1}$, we have 


$$
\begin{aligned}
\left\|a_{(\mu)} u_{p+\mu, q}\right\|^{2} & =\left\|a_{(\mu)} \alpha\left(D_{x}\right) u_{p+\mu, q}\right\|^{2} \\
& \leqq 2\left\|\tilde{\alpha}\left(D_{x}\right) a_{(\mu)} u_{p+\mu, q}\right\|^{2}+C_{1}\left\|\left\langle D_{x}\right\rangle u_{p+\mu, q}\right\|^{2} \\
& \leqq C_{2} n^{2}\left\{\left\|\left\langle D_{x}\right\rangle^{-1} a_{(\mu)} u_{p+\mu, q}\right\|^{2}+\left\|u_{p+\mu, q}\right\|^{2}\right\},
\end{aligned}
$$

where $C_{j}, j=1,2$, are constants independent of $n, p$ and $q$.

Now, in view of (A.1), the latter inequality of (ii) is proved.

(iii) It follows from $a_{(\mu)}^{(\nu)} \in S_{1,0}^{2-|\nu|}$ that

$$
\begin{aligned}
\left|I_{4}\right| \leqq L^{-1}(\log n)^{-2} \text { const. } & \sum_{2 \leqq|\nu+\mu| \leqq N_{0}}\left\|\left\langle D_{x}\right\rangle^{2-|\nu|} u_{p+\mu, q+\nu}\right\|^{2}+L(\log n)^{2}\left\|u_{p, q}\right\|^{2} \\
& \leqq L^{-1}(\log n)^{-2} \text { const. } \\
& \sum_{2 \leqq|\nu+\mu| \leqq N_{0}} n^{2(2-|\nu|)}\left\|u_{p+\mu, q+\nu}\right\|^{2}+L(\log n)^{2}\left\|u_{p, q}\right\|^{2},
\end{aligned}
$$

where const.s are independent of $L, n, p$ and $q$.

Now, in view of (A.2), the assertion of (iii) is proved. (Observe that $K^{-1} n \leqq$ $\sqrt{1+|\xi|^{2}} \leqq K \cdot n$ for $\xi \in \operatorname{supp}\left[\alpha_{n}^{(p)}\right]$.)

(Step 3.) Let us now observe that $c_{p, q}^{n}(\log n)^{-1}=M c_{p, q+\nu}^{n}$ for $|\nu|=1, c_{p, q}^{n} n(\log n)^{-1}$ $=M c_{p+\mu, q}^{n}$ for $|\mu|=1$, and $c_{p, q}^{n}(\log n)^{-2} n^{2-|\nu|} \leqq M^{|\nu+\mu|} c_{p+\mu, q+\nu}^{n}$ for $|\nu+\mu| \geqq 2$ (when $n$ is large). Therefore, it follows from (2.3) and Lemma 2 that

$$
\begin{aligned}
& \frac{d}{d t}\left\|c_{p, q}^{n} u_{p, q}\right\|^{2} \\
& \quad \leqq 2\left\{-\operatorname{Re}\left(a c_{p, q}^{n} u_{p, q}, c_{p, q}^{n} u_{p, q}\right)+\right. \\
& \quad+\left\{2+3 L(\log n)^{2}\right\}\left\|c_{p, q}^{n} u_{p, q}\right\|^{2}+ \\
& \quad+L^{-1} \text { const. } M^{2 N_{0}} \sum_{1 \leqq} \sum_{\nu+\mu \mid \leqq N_{0}}\left(\operatorname{Re}\left(a c_{p+\mu, q+\nu}^{n} u_{p+\mu, q+\nu}, c_{p+\mu, q+\nu}^{n} u_{p+\mu, q+\nu}\right)\right. \\
& \left.\left.\quad+\left\|c_{p+\mu, q+\nu}^{n} u_{p+\mu, q+\nu}\right\|^{2}\right)\right\}+ \text { negligible terms. }
\end{aligned}
$$

Let us take $L$ sufficiently large and sum up the inequalities (2.5) with respect to $(p, q)$ satisfying $|p+q| \leqq N_{n}-N_{0}$. Then, the third terms on the right hand side of (2.5) will be absorbed into the first ones, that is, we have (also observe that the terms $\frac{d}{d t}\left\|c_{p, q}^{n} u_{p, q}\right\|^{2}$ and $\operatorname{Re}\left(a c_{p, q}^{n} u_{p, q}, c_{p, q}^{n} u_{p, q}\right),(p, q)$ satisfying $N_{n}-N_{0} \leqq|p+q| \leqq N_{n}$, are negligible)

$$
\begin{aligned}
\frac{d}{d t} S_{n}^{M}(u(\cdot, t)) & \leqq-\sum_{|p+q| \leqq N_{n}} \operatorname{Re}\left(a c_{p, q}^{n} u_{p, q}, c_{p, q}^{n} u_{p, q}\right) \\
& +2\left\{2+3 L(\log n)^{2}+L^{-1} \text { const. } M^{2 N_{0}}\right\} S_{n}^{M}(u(\cdot, t))+0\left(n^{-2 s}\right),
\end{aligned}
$$

where const. is independent of $M, L$ and $n$.

Furthermore, it follows from the assumption (A.2) that

$$
\frac{d}{d t} S_{n}^{M}(u(\cdot, t)) \leqq-\frac{1}{\varepsilon}(\log n)^{2} S_{n}^{M}(u(\cdot, t))+0\left(n^{-2 s}\right)
$$




$$
\leqq-\frac{1}{\varepsilon}(\log n) S_{n}^{M}(u(\cdot, t))+0\left(n^{-2 s}\right),
$$

where $\varepsilon>0$ can be chosen arbitararily small. (For any positive number $\varepsilon$, the inequality (2.7) holds when $n$ is sufficiently large.)

Therefore we have

$$
\begin{aligned}
S_{n}^{M}(u(\cdot, t)) & \leqq \exp (-(\log n) t / \varepsilon) S_{n}^{M}(u(\cdot, 0))+0\left(n^{-2 s}\right) \\
& =n^{-t / \varepsilon} S_{n}^{M}(u(\cdot, 0))+0\left(n^{-2 s}\right) .
\end{aligned}
$$

Now, the assertion of Theorem 1 is proved, because $S_{n}^{M}(u(\cdot, 0))=0\left(n^{k}\right)$ for some $k$. q.e.d.

\section{§3. Proofs of Propositions.}

Proof of Proposition 1. Here, we consider the values of $\left\|c_{p, q}^{n} \alpha_{n}^{(p)} \beta_{n(q)} u\right\|,(p$, $q)$ satisfying $|p+q| \leqq N_{n}$, when $\left(x_{0}, \xi_{0}\right) \notin \mathrm{WF}(u)$ and $r_{0}$ (size of micro-localizers) is small.

Take a function $\zeta \in C_{0}^{\infty}$ so that $\zeta(x)=1$ in $\left\{x ;\left|x-x_{0}\right| \leqq r_{0}\right\}$. Then we have $\beta_{n} \subset \subset \zeta$ (i.e. $\zeta=1$ in the support of $\beta_{n}$ ) and $\zeta u \in H^{-k}$ for some $k$. Let us write

$$
\begin{aligned}
c_{p, q}^{n} \alpha_{n}^{(p)} \beta_{n(q)} u & =c_{p, q}^{n} \alpha_{n}^{(p)} \beta_{n(q)}(\zeta u) \\
& =\sum_{|\nu| \leqq N_{0}} \nu !^{-1} c_{p, q}^{n} \beta_{n(q+\nu)} \alpha^{(p+\nu)}(\zeta u)+c_{p, q}^{n}{ }^{r^{\prime} p, q}\left(x, D_{x}\right)(\zeta u),
\end{aligned}
$$

where $N_{0}$ is a large integer whose definition will be given later (see (3.1)).

On the last terms of the right hand side, we have the following: By the same argument as in the proof of Lemma 2 (i), the values of

$$
\left\|c_{p, q}^{n} r_{p, q}^{\prime}\left(x, D_{x}\right)\left\langle D_{x}\right\rangle,{ }^{k}\right\|_{L^{2} \rightarrow L^{2}},
$$

$(p, q)$ satisfying $|p+q| \leqq N_{n}$, are estimated by $n^{-s}$ const. $(2 C / M)^{|p+q|}$ (const. is independent of $n, p$ and $q$ ), provided

$$
k-N_{0}-1 \leqq-s
$$

Therefore, if we take $M$ so that $(2 d)(2 C / M)^{2}<1 / 2$, then

$$
\begin{aligned}
& \sum_{|p+q| \leqq N_{n}}\left\|c_{p, q}^{n} r^{\prime}{ }_{p, q}\left(x, D_{x}\right)(\zeta u)\right\|^{2} \\
&=\sum_{|p+q| \leqq N_{n}}\left\|c_{p, q}^{n} r_{p, q}^{\prime}\left(x, D_{x}\right)\left\langle D_{x}\right\rangle k\left\langle D_{x}\right\rangle^{-k}(\zeta u)\right\|^{2} \\
& \leqq \text { const. } \sum_{|p+q| \leqq N_{n}}\left\|c_{p, q}^{n} r_{p, q}^{\prime}\left(x, D_{x}\right)\left\langle D_{x}\right\rangle^{k}\right\|_{L^{2} \rightarrow L^{2}}^{2} \\
& \leqq \text { const. } \sum_{q, p}(2 C / M)^{2|p+q|} n^{-2 s} \\
& \leqq \text { const. } n^{-2 s},
\end{aligned}
$$

where const.s are independent of $n$. 
On the other terms of the right hand side, we have the following: Let us observe that

$$
\left\|\beta_{n(p+\nu)} \alpha_{n}^{(p+\nu)}(\zeta u)\right\| \leqq \sup \left|\beta_{n(p+\nu)}\right| \sup \left|\alpha_{n}^{(p+\nu)}\right| \times\left\{\int_{\Lambda_{n}}|\zeta u(\xi)|^{2} d \xi\right\}^{1 / 2},
$$

where $\Lambda_{n}=\left\{\xi ;\left|\xi-n \xi_{0}\right| \leqq n r_{0}\right\}$. Therefore, it follows from the assumptions of Proposition 1 that, for any positive number $s$, there exists a constant $C^{\prime}$ (independent of $n, p$ and $q)$ such that the values of $\left\|\beta_{n(p+\nu)} \alpha^{(p+\nu)}(\zeta u)\right\|$ are estimated by $n^{-s-|p+\nu|}$ $C^{\prime}\left(C N_{n}\right)^{|p+q|}$.

Therefore, if we take $M$ so that $(2 d)(2 C / M)^{2}<1 / 2$, then

$$
\begin{aligned}
& \sum_{|p+q| \leqq N_{n}}\left\|c_{p, q}^{n} \beta_{n(p+\nu)} \alpha_{n}^{(p+\nu)}(\zeta u)\right\|^{2} \\
& \quad \leqq C_{1} \sum_{p, q}(2 C \mid M)^{2|p+q|} n^{-2 s-2|\nu|} \leqq 2 C_{1} n^{-2 s-2|\nu|,}
\end{aligned}
$$

where $C_{1}$ is a constant independent of $n$.

Combining the above arguments, we have the assertion of Proposition 1.

Proof of Proposition 2. Let $\zeta \in C_{0}^{\infty}$ be a function satisfying $\zeta \subset \subset \beta_{n}$ (i.e. $\beta_{n}=1$ in the support of $\left.\zeta\right)$. First, we shall show that $\left\|\alpha_{n}(D) \zeta u\right\|=0\left(n^{-s}\right)$ as $n^{\rightarrow \infty}$, when $S_{n}^{M}(u)=0\left(n^{-2 s}\right)$ holds. Let us consider

$$
\begin{aligned}
\left\|\alpha_{n}(D) \zeta u\right\| & =\left\|\alpha_{n}(D) \zeta\left(\beta_{n} u\right)\right\| \\
& \leqq \sum_{|\nu| \leqq N_{0}} \nu !^{-1} \sup \left|\zeta_{(\nu)}\right|\left\|\alpha_{n}^{(\nu)} \beta_{n} u\right\|+\left\|r^{\prime \prime}\left(x, D_{x}\right) \beta_{n} u\right\| .
\end{aligned}
$$

In the above inequality, we have $\left\|\alpha_{n}^{(\nu)} \beta_{n} u\right\|=0\left(n^{-s-|\nu|}(\log n)^{|\nu|}\right)$ from the hypothesis. So we must consider only the last term. Let us now take a function $\tilde{\zeta} \in C_{0}^{\infty}$ so that $\tilde{\zeta}=1$ in the support of $\beta_{n}$. Then, for some $k, \tilde{\zeta} u \in H^{-k}$. Therefore, by the same argument as in the proof of Lemma 2 (i), we can show that

$$
\begin{aligned}
\left\|r^{\prime \prime}\left(x, D_{x}\right) \beta_{n} u\right\| & =\left\|r^{\prime \prime}\left(x, D_{x}\right) \beta_{n}\left\langle D_{x}\right\rangle\left\langle D_{x}\right\rangle-k \tilde{\zeta} u\right\| \\
& =0\left(n^{-s}\right),
\end{aligned}
$$

provided $N_{0}$ satisfies the inequality $k-N_{0}-1 \leqq-s$.

Now, we remark that, for the proof of Proposition 2, it suffices to show that, for any positive number $s$,

$$
\sup \left|\alpha_{n}(\xi) \widehat{\zeta u}(\xi)\right|=0\left(n^{-s}\right) \text { as } n^{\rightarrow \infty} \text {, }
$$

from the assumption of Proposition 2. To see this, let us observe that

$$
\begin{aligned}
\left|\alpha_{n}(\xi) \widehat{\zeta u}(\xi)\right| & \leqq\left\|\alpha_{n}(D) \zeta u\right\|_{L^{1}} \\
& \leqq \text { const. } \|\left(1+|x|^{2}\right)^{l} \alpha_{n}(D) \zeta u \mid,
\end{aligned}
$$

where $l$ is a integer satisfying $l>d / 2$. Furthermore,

$$
\left(1+|x|^{2}\right)^{l} \alpha_{n}(D)(\zeta u)
$$




$$
\begin{aligned}
& =\sum_{|\kappa| \leqq 2 l}(-1)^{|\kappa|} \mid \kappa !^{-1} \alpha_{n}^{(\kappa)}\left[\left(-i \partial_{x}\right)^{\kappa}\left(1+|x|^{2}\right)^{l}\right](\zeta u) \\
& =\sum_{|\kappa| \leqq 2 l} \alpha_{n}^{(\kappa)} \zeta_{\kappa} u,
\end{aligned}
$$

where we denote $\zeta_{\kappa}=(-1)^{|\kappa|} \kappa !^{-1}\left[\left(-i \partial_{x}\right)^{\kappa}\left(1+|x|^{2}\right)^{l}\right] \zeta$.

Thus we have

$$
\left|\alpha_{n}(\xi) \widehat{\zeta u}(\xi)\right| \leqq \text { const. } \sum_{\kappa \mid \leqq 2 l}\left\|\alpha_{n}^{(\kappa)} \zeta_{\kappa} u\right\|
$$

In the last inequality, we can show that, for any positive number $s,\left\|\alpha_{n} \zeta_{\kappa} u\right\|=$ $0\left(n^{-s-|\kappa|}(\log n)^{|\kappa|}\right)$ by the same argument as in the forepart of the proof, which completes the proof.

\section{§4. Appendix (Proof of Theorem 2).}

The proof of Theorem 2 is quite analogeous to that of Theorem 1. We shall show that, provided $a\left(x, D_{x}\right) u=f$ and $a\left(x, D_{x}\right)$ satisfies the condition (A.3), $S_{n}^{M}(f)$ $=0\left(n^{-2 s}\right)$ implies $S_{n}^{M}(u)=0\left(n^{-2 s}\right)$ for any given positive number $s$.

Let us first operate $\alpha_{n}^{(p)}(D) \beta_{n(q)}(x),(p, q)$ satisfying $|p+q| \leqq N_{n}$, to the both sides of the equation $a\left(x, D_{x}\right) u=f$. Then

$$
\alpha_{n}^{(p)} \beta_{n(q)} a u=\alpha_{n}^{(p)} \beta_{n(q)} f .
$$

This implies

$$
a u_{p, q}+\sum_{0<|\nu+\mu| \leqq N_{0}} \frac{(-1)^{|\nu|}}{\nu ! \mu !} a_{(\mu)}^{(\nu)} u_{p+\mu, q+\nu}+r_{q, q} u=f_{p, q} .
$$

Here we have used the same notations as in the proof of Theorem 1. Therefore we have

$$
\begin{aligned}
& \left\|a u_{p, q}\right\|^{2} \\
& \qquad \text { const. }\left\{\sum_{0<|\nu+\mu|<m}\left\|a_{(\mu)}^{(\nu)} u_{p+\mu, q+\mu}\right\|^{2}+\sum_{m \leqq|\nu+\mu| \leqq N_{0}}\left\|a_{(\mu)}^{(\nu)} u_{p+\mu, q+\nu}\right\|^{2}\right. \\
& \left.\quad+\left\|r_{p, q} u\right\|^{2}+\left\|f_{p, q}\right\|^{2}\right\} \\
& =\text { const. }\left\{J_{1}+J_{2}+\left\|r_{p, q} u\right\|^{2}+\mid f_{p, q} \|^{2}\right\},
\end{aligned}
$$

where const. means a constant independent of $n, p$ and $q$.

Almost the same arguments as in the proof of Lemma 2 yield the following:

Lemma 3. (i) For any positive number s, there exists a positive integer $N_{0}$ such that

$$
\sum_{|p+q| \leqq N_{n}}\left\|c_{p, q}^{n} r_{p, q} u\right\|^{2}=0\left(n^{-2 s}\right) \text { as } n \rightarrow \infty .
$$

(ii) For any positive number $\varepsilon$, there exists a constant $C_{\varepsilon}$ such that

$$
J_{1} \leqq \sum_{0<|\nu+\mu|<m}\left\{(\log n)^{-|\nu+\mu|} n^{|\mu|}\right\}^{2}\left\{\varepsilon\left\|a u_{p+\mu, q+\nu}\right\|^{2}+C_{\varepsilon}\left\|u_{p+\mu, q+\nu}\right\|^{2}\right\},
$$


and

$$
J_{2} \leqq \sum_{m \leqq|\nu+\mu| \leqq N_{0}}\left\{(\log n)^{-m} n^{m-|\nu|}\right\}^{2}\left\{\varepsilon\left\|a u_{p+\mu, q+\nu}\right\|^{2}+C_{\varepsilon}\left\|u_{p+\mu, q+\nu}\right\|^{2}\right\} .
$$

Let us continue the proof of Theorem 2. Observe now that $(\log n)^{-|\nu+\mu|} n^{|\mu|} c_{p, q}^{n}$ $=M^{|\nu+\mu|} c_{p+\mu, q+\nu}^{n}$ and, for $(\nu, \mu)$ satisfying $|\nu+\mu| \geqq m, \quad(\log n)^{-m} n^{m-|\nu|} c_{p, q}^{n} \leqq M^{|\nu+\mu|}$ $c_{p+\mu, q+\nu}^{n}$ (when $n$ is large). So, in view of (4.1) and Lemma 3, we have

$$
\begin{aligned}
& \left\|a\left(c_{p, q}^{n} u_{p, q}\right)\right\|^{2} \\
& \leqq \text { const. } \quad M^{2 N_{0}}\left\{\varepsilon \sum_{0<|\nu+\mu| \leqq N_{0}}\left\|a\left(c_{p+\mu, q+\nu}^{n} u_{p+\mu, q+\nu}\right)\right\|^{2}+C_{\varepsilon}\left\|c_{p+\mu, q+\nu}^{n} u_{p+\mu, q+\nu}\right\|^{2}\right\} \\
& \quad+\text { negligible terms. }
\end{aligned}
$$

Let us choose $\varepsilon>0$ sufficiently small and sum up the both sides of (4.2) with respect to $(p, q)$ satisfying $|p+q| \leqq N_{n}-N_{0}$. Then, the first terms on the right hand side of (4.2) will be absorbed into the left hand side, that is, we have (also observe that $\left\|a\left(c_{p, q}^{n} u_{p, q}\right)\right\|^{2}$ with $(p, q)$ satisfying $N_{n}-N_{0} \leqq|p+q| \leqq N_{n}$ are negligible)

$$
\sum_{|p+q| \leqq N_{n}}\left\|a\left(c_{p, q}^{n} u_{p, q}\right)\right\|^{2} \leqq \text { const. } S_{n}^{M}(u)+0\left(n^{-2 s}\right) .
$$

In view of the condition (A.3), it is clear that

$$
(\log n)^{2 m} S_{n}^{M}(u) \leqq \text { const. } \sum_{|p+q| \leqq N_{n}}\left\|a\left(c_{p, q}^{n} u_{p, q}\right)\right\|^{2},
$$

when $n$ is large. Therefore, it follows from (4.3) that

$$
S_{n}^{M}(u)=0\left(n^{-2 s}\right) \text { as } n \rightarrow \infty,
$$

which completes the proof.

q.e.d.

\section{Department of Mathematics Kyoto University}

\section{References}

[1] L. Hörmander, The analysis of linear partial differential operators III, Springer Verlag, 1985.

[2] T. Hoshiro, A property of operators characterized by iteration and a necessary condition for hypoellipticity, J. Math. Kyoto Univ 27 (1987) 401-416.

[3] S. Kusuoka and D. Strook, Applications of the Malliavin calculus, part II, J. Fac. Sci. Univ. Tokyo Sect. IA, Math., 32 (1985), 1-76.

[4] S. Mizohata, On the Cauchy problem, Lectures delivered at the Wuhan University, Academic Press (Notes and Reports in Math. in Science and Engineering Vol. 3) 1986.

[5] S. Mizohata, On analytic regularities, Séminaire de Vaillant à Paris VI, (1984).

[6] Y. Morimoto, Non-hypoellipticity for degenerate elliptic operators, Publ. RIMS Kyoto Univ., 22 (1986) 25-30.

[7] Y. Morimoto, A criterion for hypoellipticity of second order differential operators, preprint.

[8] Y. Morimoto, Criteria for hypoellipticity of differential operators, part II, Publ RIHS Kyoto Univ. 22 (1986) 1129-1154. 